\title{
Piezoelectric polymer actuators for active vibration isolation in space applications
}

\section{Authors: G. Bohannan, V. Hugo Schmidt, D. Brandt, and M. Mooibroek}

NOTICE: this is the author's version of a work that was accepted for publication in Ferroelectrics. Changes resulting from the publishing process, such as peer review, editing, corrections, structural formatting, and other quality control mechanisms may not be reflected in this document. Changes may have been made to this work since it was submitted for publication. A definitive version was subsequently published in Ferroelectrics, [VOL\# 224, (1999)] DOI\# 10.1080/00150199908210569

G. Bohannan, V. Hugo Schmidt, D. Brandt, and M. Mooibroek, "Piezoelectric polymer actuators for active vibration isolation in space applications," Ferroelectrics 224, 211-217 (1999). http://dx.doi.org/10.1080/00150199908210569

Made available through Montana State University's $\underline{\text { ScholarWorks }}$ scholarworks. montana.edu 


\title{
Piezoelectric Polymer Actuators for Active Vibration Isolation in Space Applications
}

\author{
G. BOHANNAN, H. SCHMIDT, D. BRANDT and M. MOOIBROEK
}

Montana State University, Bozeman, MT, 59717, USA

(Received August 24, 1998; In final form September 14, 1998)

\begin{abstract}
A lightweight actuator for active vibration isolation in space applications is being developed to replace the heavy electromagnetic systems now in use. The actuator has a low effective spring constant that provides for passive vibration damping down to sub-Hertz frequencies while allowing the isolated experiment to follow the near-dc bias motion of the spacecraft. The actuator is currently optimized for the vibration level of the Space Shuttle and assembled from a pair of bimorphs in a leaf-spring configuration. Changing the size and number of sheets used in construction can vary electromechanical properties. Passive damping has been demonstrated in one and two-dimensional tests. For large (greater than a few kilograms) suspended masses, the system is underdamped and relative velocity feedback must be used to remove the resonance. Real-time control of the resonance frequency is achieved by controlling the voltage applied to the actuator with feedback from a displacement sensor. A folded pendulum seismic monitoring device was adapted for use as a one-dimensional low frequency test platform and has obtained accurate measurements of the effective spring constant and damping coefficient. Single-degree-of-freedom active feedback testing is also being conducted using this device. Two-dimensional (three-degree-of-freedom) passive damping tests were conducted on NASA's KC-135 Reduced Gravity Platform in March 1998.
\end{abstract}

Keywords: piezoelectric polymers; vibration isolation; actuators

\section{INTRODUCTION}

An opportunity to demonstrate practical applications of piezoelectric polymers arose with the challenge of replacing the heavy electromagnetic systems now in use on the Space Shuttle for vibration isolation. The goal of 
this project has been to develop a lightweight, low-power system for use in near zero-g applications. By taking advantage of the properties of a springlike device with a low natural spring constant, passive damping down to low frequencies can be used. Active feedback is used to dampen the low frequency resonance.

\section{DESIGN REQUIREMENTS}

In the low gravity environment of the Space Shuttle, a very large experiment (10's to 100's of kilograms) can be suspended for vibration isolation using devices exerting very small forces. Small bias forces resulting in one to three micro-g arise due to the fact that the experiments to be isolated are not at the center of gravity of the spacecraft. Vibrations, referred to as g-jitter, occur across the frequency spectrum from sub-Hertz to kilohertz. These vibrations measure in the tens to several hundred micro-g. Pulse noise levels produce accelerations on the order of 1000 micro-g, but these contain very high frequency components and passive damping eliminates their effect.

Modeling has indicated that, for suspended loads greater than a few kilograms, passive damping can provide isolation for frequencies above a few Hertz. Active damping is added to remove natural resonance. The low force requirement creates an additional requirement for relatively large displacement. For the Space Shuttle environment, a relative displacement of $+/$ - one centimeter is required to achieve a less than 10 micro-g loading on the isolated box. This relative displacement grows with the vibration level of the platform and would be almost a meter for the same level of isolation on an aircraft such as NASA's KC-135 Reduced Gravity Platform.

\section{CONSTRUCTION}

The actuators resemble a small leaf spring. The upper and lower halves of the actuator use a bimorph layout; each bimorph consists of cantilevered-beam sections. Silver is deposited on PVDF sheets to form the necessary electrode pattern on the top and bottom of each layer. Adhesives are used to bond the layers to form the bimorph and to bond the edges of the two bimorphs to make the leaf spring. The bimorph pre-curvature is obtained by placing the two sheets in a press while the adhesive sets. Copper tabs are attached to the edges to allow routing of control voltages onto the electrode layers. Exposed electrode layers are kept at ground potential for safety. Plus and minus voltages are applied to obtain the appropriate "s" curvature. 
In the configuration shown in Figure 1, the voltage polarities cause the actuator to expand. Opposite polarities would produce contraction. Stacking actuators in series allows a larger displacement for the same applied voltage.

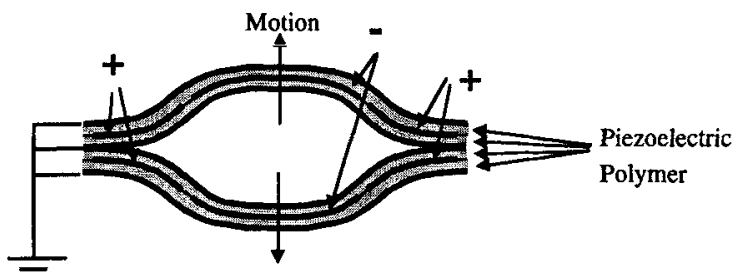

FIGURE 1 Schematic end view of the actuator. Exposed electrodes are grounded, inner electrode layers are charged with opposite polarity to achieve proper curvature.

\section{ELECRICAL AND MECHANICAL TESTING}

The low force, large displacement characteristics present a challenge for testing. A seismic monitoring device with near zero-g restoring force in one dimension was adapted for use as a test platform. The device, shown in figure 2 , consists of a platform suspended between a normal pendulum arm and an inverted pendulum arm ${ }^{[1]}$. The tuning mass and platform have a combined mass of approximately $20-\mathrm{kg}$. Additional mass may be added for finer tuning. The restoring force from the normal arm is almost canceled by the antirestoring force of the inverted arm. By adjusting the lengths of the arms and the position of the tuning mass it is possible to achieve periods of greater than 20 seconds, or restoring forces of less than 10 micro-g per millimeter of motion. This residual force is small enough that the parameters of the actuator can be readily extracted.

Parameters of actuators with dimensions of $5.0 \times 5.0 \times 0.5 \mathrm{~cm}^{3}$ have been measured dynamically using the folded pendulum. Typical values for the passive spring constant are between 15 to $20 \mathrm{~N} / \mathrm{m}$ and passive damping constants are between 1.3 to $1.6 \mathrm{~kg} / \mathrm{sec}$. The variation is due primarily to an inconsistency in construction technique, e.g. controlling the thickness of the adhesive layer. Static measurements show the no-load displacement is approximately $3 \mathrm{~mm}$ per kilovolt.

The suspended platform provides a near zero-g environment in one dimension, partially simulating the isolated experiment on the Space Shuttle. Data obtained from Shuttle micro-gravity missions contain greater than 10 


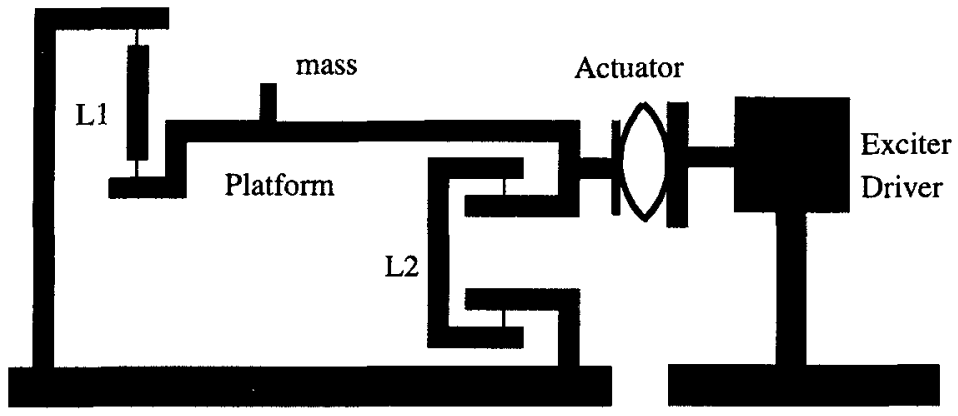

FIGURE 2 Schematic of the folded pendulum test platform. L1 is the normal pendulum arm. L2 is the inverted pendulum arm.

micro-g biases, so this test system is well within the error budget of available data. The platform is coupled via the actuator to an exciter driver that simulates the vibrating shuttle frame. A low-frequency electronic oscillator controls the exciter driver. Position and accelerometer sensor data is wired into a multifunction board in a laboratory PC for data analysis.

\section{ACTIVE DAMPING IN ONE DIMENSION}

Tests conducted using the folded pendulum demonstrated that displacement feedback could be used to change the resonance frequency and induce active damping. An optical position sensing device (PSD) was used to measure the relative displacement between the platform and the exciter/driver. Application of a voltage in-phase with the relative displacement reduced the effective actuator spring constant, while application of voltage out-of-phase with the displacement effectively stiffened the actuator. Measurements of resonant frequency shifts confirmed the adjustment in the effective spring constant.

When accelerometer feedback was used to produce a force opposite to the direction of acceleration, measurable damping was observed at frequencies of $5 \mathrm{~Hz}$ and above. However, at lower frequencies the signal from the accelerometer dropped below the device noise and damping was not as effective as predicted by modeling.

One notable observation made while using the folded pendulum is that the tuning mass may be adjusted to the point that without the actuator in 
place, the pendulum would soon become unstable. This slight "negativetuning" appeared to improve the phase accuracy of dynamic measurements.

\section{PASSIVE DAMPING ON THE KC-135}

NASA has recently been offering opportunities for undergraduates to conduct experiments on their KC-135A Reduced Gravity Platform aircraft. This project was selected for a pair of flights that occurred in March 1998. The KC-135A achieves near zero-g conditions for up to about 25 seconds at a time by flying a series of parabolic loft maneuvers. Each flight typically consists of 40 parabolic maneuvers. The g-jitter experienced by the aircraft is approximately 100 times greater than that of the space shuttle, but a few relatively quiet periods on the order of a few seconds in duration were experienced during each the two flights. The difficulties with the aircraft include air turbulence not felt by the shuttle and the fact that the pilots manually control the aircraft during the maneuvers.

The experiment consisted of a suspended mass with three degrees-offreedom (two translational, one rotational) shown in figure 3. Eight sets of two stacked actuators were used. The suspended mass used in this test was less than two $\mathrm{kg}$, which was much smaller than anticipated masses to be isolated on the Space Shuttle. With the higher vibration level, a stiffer system with much faster relaxation time was required. The stiffer system was also necessary to constrain the suspended mass to within the volume of the experiment rack. Even with the stiffening, edge padding was required to constrain the mass during turbulent periods.

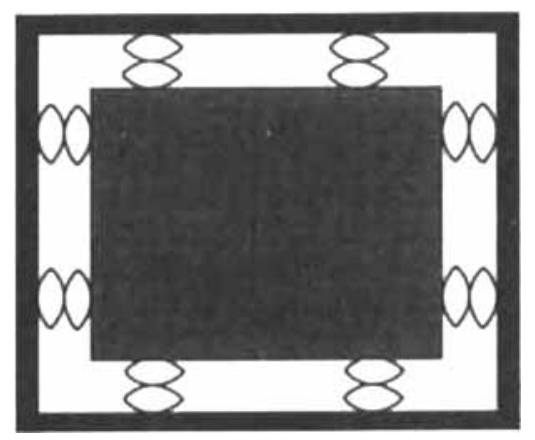

FIGURE 3 Schematic of the three-degree-of-freedom experiment flown on NASA's KC-135 Reduced Gravity Platform. 
Due to potential electrical safety problems arising from the separation of the bimorphs, it was necessary to disconnect the actuators from the driver amplifiers and test only the passive characteristics of the system. The accelerometer and position sensing devices remained in operation and control signals were monitored during flight for post facto review of how well the prototype control circuitry operated.

Data obtained from the flight indicate that the actuators did suspend the simulated experiment box and provide passive damping for up to several seconds at a time. These periods were too short to obtain accurate measurements of time constants, so the information gained was merely qualitative.

\section{PLANNED IMPROVEMENTS}

One of the major problems with the actuators was that a high percentage of them experienced adhesive failure along the edge joint between the upper and lower bimorph. Since PVDF is a relative of TEFLON, it is not surprising that adhesives are problematic. Alternative bonding techniques, including several types of welding are now being investigated.

Higher force levels can theoretically be achieved by increasing the number of layers used, i.e. instead of a two-layer, a "multi-morph" configuration will be used. Progress along this line will depend on the results of the bonding tests.

As an alternative to using an accelerometer for deriving the lowfrequency feedback signal, use of a differential circuit applied to the position sensing device signal is being investigated. The difficulty to be overcome here is due to the fact that the differentiated relative position signal is not a direct readout of forces on the suspended box.

\section{CONCLUSION}

The one-dimensional tests have shown that the actuator response matches the predicted response and that the feedback from acceleration and position sensors can be used to induce damping. Flight-testing of a multidimensional system demonstrated that a complete system could be assembled in the volume currently allocated for this purpose while significantly reducing the weight from that of current systems. The experiments that have been completed have shown that these actuators are a viable replacement for the large electromagnets currently being used on the shuttle. Work is ongoing to improve and refine what has been developed to date. 


\section{ACKNOWLEDGEMENTS}

This work has been supported by NASA EPSCoR Grant NCCW-0058 and by the Montana Space Grant Consortium.

\section{References}

[1] D. G. Blair, J. Liu, E. F. Moghaddam, L. Ju, Physics Letters A 193 (1994) 223-226 